\title{
Reflection, Rewinding, and Coin-Toss in EasyCrypt
}

\author{
Denis Firsov \\ deins@cs.ioc.ee \\ Tallinn University of Technology \\ Estonia \\ Guardtime \\ Tallinn, Estonia
}

\author{
Dominique Unruh \\ unruh@ut.ee \\ University of Tartu \\ Estonia
}

\begin{abstract}
In this paper we derive a suite of lemmas which allows users to internally reflect EasyCrypt programs into distributions which correspond to their denotational semantics (probabilistic reflection). Based on this we develop techniques for reasoning about rewinding of adversaries in EasyCrypt. (A widely used technique in cryptology.) We use our reflection and rewindability results to prove the security of a coin-toss protocol.
\end{abstract}

\section{CCS Concepts: • Security and privacy $\rightarrow$ Logic and ver- ification.}

Keywords: cryptography, formal methods, EasyCrypt, reflection, rewindability, commitments, binding, coin-toss

\section{ACM Reference Format:}

Denis Firsov and Dominique Unruh. 2022. Reflection, Rewinding, and Coin-Toss in EasyCrypt. In Proceedings of the 11th ACM SIGPLAN International Conference on Certified Programs and Proofs (CPP '22), January 17-18, 2022, Philadelphia, PA, USA. ACM, New York, NY, USA, 14 pages. https://doi.org/10.1145/3497775.3503693

\section{Introduction}

Handwritten cryptographic security proofs are inherently error-prone. Humans will make mistakes both when writing and when checking the proofs. To ensure high confidence in cryptographic systems, we use frameworks for computeraided verification of cryptographic proofs. One widely used such framework is the EasyCrypt tool [5]. In EasyCrypt, a cryptographic proof is represented by a sequence of "games" (simple probabilistic programs), and the relationship between programs are analyzed in a probabilistic relational Hoare logic (pRHL). EasyCrypt has been successfully used to verify a variety of cryptographic schemes: electronic voting [13], digital signatures [14], differential privacy [3], security of IPsec [16], and many others.

Permission to make digital or hard copies of part or all of this work for personal or classroom use is granted without fee provided that copies are not made or distributed for profit or commercial advantage and that copies bear this notice and the full citation on the first page. Copyrights for thirdparty components of this work must be honored. For all other uses, contact the owner/author(s).

CPP '22, January 17-18, 2022, Philadelphia, PA, USA

(c) 2022 Copyright held by the owner/author(s).

ACM ISBN 978-1-4503-9182-5/22/01.

https://doi.org/10.1145/3497775.3503693
However, there is one cryptographic proof technique that seems very difficult to implement in EasyCrypt, namely rewinding. Rewinding is ubiquitous in more advanced proofs, especially when involving zero-knowledge proofs, multiparty computation, but also in relatively simple cases such as building a coin-toss from a commitment. (The latter case we will explore in Sec. 5.) In a nutshell, rewinding refers to the proof technique in which we take a given (usually unknown) program $A$ (an adversary), and convert it into an adversary $B$ that performs the following or similar steps:

1. Remember the initial state of $A$.

2. Run $A$.

3. Restore the original initial state of $A$.

4. Run $A$ again.

5. Combine the results from the runs and/or repeat this until it yields a desired outcome.

While the above steps seem simple, we run into numerous challenges when trying to implement rewinding in EasyCrypt, both due to restrictions in the type system, and due to the necessity for reasoning about probability distributions of program outputs in a way that is not directly supported by EasyCrypt's tactics.

To the best of our knowledge, rewinding has not been implemented in EasyCrypt, nor in other frameworks for reasoning about cryptographic proofs, see Sec. 1.4.

Our contribution. In this work, we design a set of tools to address rewindability in the EasyCrypt framework, and for reasoning about the probabilistic semantics of programs inside EasyCrypt (we call this probabilistic reflection). We validate our results by developing a formal proof of a cointoss protocol based on rewinding. The EasyCrypt code of our framework is found in [15].

\subsection{Challenges}

To understand the motivation behind our project, let us look at the example of a pen-and-paper derivation using rewinding. When analyzing a coin-toss protocol based on a commitment, we are faced with the following situation: We have an adversary $A$ that can open the commitment to contain given boolean value $b$ (with some non-zero probability). We want to show that this means that $A$ could also, in the same run, produce openings to both boolean values (i.e., false and true). This is done by defining a different adversary $B$ that runs A.commit (to produce the commitment), stores the state of $A$, 
runs A.open(false) (to produce the first opening), restores the state of $A$, and runs A.open(true) (to produce the second opening). Then we show that the probability that $B$ produces two valid openings is lower-bounded in terms of the probability that $A$ is successful in producing one valid opening. As we will see in more detail in Sec. 5.1, the core theorem for showing this is the following.

Theorem 1.1. Let $A$ be a probabilistic program and let $\boldsymbol{m}$ denote a memory configuration which represents an initial state of $A$. We write $\operatorname{Pr}[r \leftarrow X . p() @ \boldsymbol{m}: M]$ to denote the probability of a predicate $M$ being satisfied by the result of running the procedure $p()$ of a module $X$ on the initial memory $\boldsymbol{m}$. In this case, the following inequality holds:

$$
\begin{aligned}
& \operatorname{Pr}\left[\begin{array}{c}
\text { A.init(); s } \leftarrow \text { A.getState(); } \\
r_{1} \leftarrow \text { A.main();A.setState }(s) ; \\
r_{2} \leftarrow \text { A.main()@ } \boldsymbol{m}: r_{1} \wedge r_{2}
\end{array}\right] \\
& \geq \operatorname{Pr}[\text { A.init(); } r \leftarrow \text { A.main()@ } \boldsymbol{m}: r]^{2} \text {. }
\end{aligned}
$$

Proof. Step (1) applies "the averaging technique" by representing $A$.init() as a family of distributions $D_{A}^{\mathrm{m}}$. ( $D_{A}^{\mathrm{m}}$ denotes the distribution in the family corresponding to memory $\mathbf{m}$.) We write $\mu_{1}\left(D_{A}^{\mathbf{m}}, \mathbf{n}\right)$ for the probability that $D_{A}^{\mathbf{m}}$ assigns to memory configuraion $\mathbf{n}$. Then $\mu_{1}\left(D_{A}^{\mathbf{m}}, \mathbf{n}\right)$ is the probability of A.init () terminating in the memory state $\mathbf{n}$ given that it starts in the initial state $\mathbf{m}$. The rest of the computations are run starting from memory configuration $\mathbf{n}$.

$$
\begin{aligned}
& \operatorname{Pr}\left[\begin{array}{c}
\text { A.init }() ; s \leftarrow \text { A.getState }() ; \\
r_{1} \leftarrow \text { A.main();A.setState }(s) ; \\
r_{2} \leftarrow \text { A.main() @ m }: r_{1} \wedge r_{2}
\end{array}\right] \\
& \stackrel{(1)}{=} \sum_{\mathbf{n}} \mu_{1}\left(D_{A}^{\mathbf{m}}, \mathbf{n}\right) \cdot \operatorname{Pr}\left[\begin{array}{c}
s \leftarrow \text { A.getState }() ; \\
r_{1} \leftarrow \text { A.main();A.setState }(s) ; \\
r_{2} \leftarrow \text { A.main()@ } \mathbf{n}: r_{1} \wedge r_{2}
\end{array}\right] \\
& \stackrel{(2)}{=} \sum_{\mathbf{n}} \mu_{1}\left(D_{A}^{\mathbf{m}}, \mathbf{n}\right) \cdot \operatorname{Pr}[r \leftarrow \text { A.main() @ } \mathbf{n}: r]^{2} \\
& \stackrel{(3)}{\geq}\left(\sum_{\mathbf{n}} \mu_{1}\left(D_{A}^{\mathbf{m}}, \mathbf{n}\right) \cdot \operatorname{Pr}[r \leftarrow \text { A.main() @ } \mathbf{n}: r]\right)^{2} \\
& \stackrel{(4)}{=} \operatorname{Pr}[\text { A.init }() ; r \leftarrow \text { A.main()@ m }: r]^{2} .
\end{aligned}
$$

Step (2) makes use of the fact that the probability of a success (i.e., A.main returning true) in both of two independent runs equals to the square of a probability of a success in a single run. Step (3) is an application of Jensen's inequality. The final step undoes the averaging.

There are number of challenges in performing this proof formally in EasyCrypt:

1. Our proof turns the program A.init() into a parameterized distribution of final memories (memories after A.init() terminates). While EasyCrypt has a notion $\operatorname{Pr}[$ A.init()@ m : $M]$ it does not formally recognize that this defines a distribution, not withstanding the suggestive syntax.

2. Also, our proof makes use of results about probability distributions (e.g., Jensen's inequality) which can be easily stated and proved in terms of probabilistic distributions while they would be much harder (or even impossible) to express and prove directly using program logics (e.g., probabilistic Hoare logic).

3. Another challenge is that EasyCrypt does not have a type "memory"; we cannot define a distribution over memories because we cannot even assign it a type. In EasyCrypt, memories are recognized purely syntactically by prepending the variable names with $\&$.

4. Finally, it is not immediate how one can generically specify the interface of programs (modules) which can return their own state. In other words, what should be the type of the return value of the function A.getState()?

\subsection{Probabilistic Reflection}

As explained above, one of the challenges is that we cannot access the distribution which corresponds to the semantics of the program. To enable this, we introduce a suite of lemmas which allows us to access the distribution corresponding to a program.

This turned out to be a powerful tool for rewinding proofs, but we also believe that it can be useful when one needs to derive facts for programs based on their denotational semantics. For example, in a situation when a particular tactic is not available in EasyCrypt, but in a pen-and-paper proof one would show it simply based on probability theory reasoning (e.g., Jensen's inequality, averaging). In the following, we sketch our solution for what we call probabilistic reflection.

(A word on terminology: The term reflection is used in many different ways in the literature. For example, in programming languages it often refers to the ability of a program to see its own structure at runtime. In proof assistants, reflection often means that one translates a term into abstract syntax and can reason about it inside the logic. Our probabilistic reflection is different from these, but we believe the term "reflection" is still justified because it allows us to expose the internal denotational semantics inside the program logic.)

Recall that there are no valid types which refer to distribution of final memories. These would be needed to give a type to the denotational semantics of a program, let alone define those semantics. However, in EasyCrypt each program has an associated variable $\mathcal{G}_{A}^{\mathbf{m}}$ (the type of $\mathcal{G}_{A}^{\mathbf{m}}$ is $\mathcal{G}_{A}$ ) which refers to the part of the memory $\mathbf{m}$ accessible by module $A$. It is guaranteed that running a program $A$ will never change anything outside $\mathcal{G}_{A}^{\mathbf{m}}$. So "effectively", the semantics of a program can be described by looking only at the $\mathcal{G}_{A}$-part of a memory. So, we define a family of distributions $D_{A}^{g}$ for $g$ of type $\mathcal{G}_{A}$ such that $\mu_{1}\left(D_{A}^{g}, h\right)$ is the probability that we get $h$ 
in $\mathcal{G}_{A}$-part of the final memory configuration when starting with $g$ in $G_{A}$-part of the initial memory configuration.

Another problem is that EasyCrypt forbids to refer to the type $\mathcal{G}_{A}$ in the top-level definitions (global definitions of operators/constants). So, in particular, we cannot define a distribution $D_{A}^{g}$ parameterized by $\mathcal{G}_{A}$-values in EasyCrypt. In our workaround to this problem, we prove lemmas of the existence of that family of distributions, but we do not define a constant referring to that family. This works since we only need to refer to the type of $D_{A}^{g}$ locally in the theorem statement. Then, when reasoning, one can inside a proof refer to "the" distribution that exists by our lemmas.

In conclusion, our lemma for probabilistic reflection looks roughly as follows:

Theorem 1.2. For all memories $\boldsymbol{m}$ and programs $A$ there exists a family of distributions $D_{A}^{g}$ (with $g$ of type $G_{A}$ ) such that for all predicates $M$ on values of type $\mathcal{G}_{A}$ :

$$
\operatorname{Pr}\left[A \cdot \operatorname{main}() @ \boldsymbol{m}: M\left(\mathcal{G}_{A}^{\text {fin }}\right)\right]=\mu\left(D_{A}^{\mathcal{G}_{A}^{m}}, M\right)
$$

Here, fin is the final memory after execution of $A$.main() and $\mu(d, M)$ denotes the probability that the predicate $M$ holds for values distributed according to $d$. Actual reflection lemma adds generality, e.g., referring also to the inputs/outputs of A.main, see Sec. 3.1.

However, being able to reflect the distribution corresponding to a given program is not enough. If we want to reason about composite programs, we will also need to understand how the different constructs in our language operate on the distributions. For example, given a program $A ; B$, reflection gives us distributions $D_{A B}, D_{A}$, and $D_{B}$ relating to the semantics of $(A ; B), A$, and $B$, respectively. However, we do not, a priori, know how $D_{A B}$ is related to $D_{A}$ and $D_{B}$. It is not even a priori clear whether inside the logic of EasyCrypt, it is possible to derive that relationship. Thus we prove additional lemmas for this and other cases that allow us to derive the distribution of a more complex program from the distributions of its components (see Sec. 3.3). For example, $D_{A B}$ is shown to be the monadic bind of $D_{A}$ and $D_{B}$.

Altogether this gives us a library for probabilistic reflection in EasyCrypt, independent of the results on rewinding below. See Sec. 3 for details.

\subsection{Rewinding}

The final challenge in the formal derivation of Thm. 1.1 is that in EasyCrypt, we cannot define a generic interface of modules which return their own state. Morally, we want A.getState() to return a value $\mathcal{G}_{A}^{\mathbf{m}}$ of type $\mathcal{G}_{A}$. However, this is impossible since the type $\mathcal{G}_{A}$ is only allowed to appear in the logical statements and program code of other modules but not in the code of the module $A$ itself.

We solve the above problem by defining what it means for a module to be rewindable. In essence, a module is rewindable if and only if the state of the program can be encoded as a bitstring (or equivalently, as any other countable type). In particular, a program with variables of type "real" (which is uncountable) would not be rewindable in that sense. ${ }^{1} \mathrm{~A}$ security proof using rewinding would then only apply to rewindable adversaries which is not a restriction from the cryptographic point of view. (Typically, cryptographic adversaries are assumed to operate on data that is representable in a computer. Such data can always be encoded as a bitstring.)

In conclusion, our definition for rewindable modules (programs) roughly requires a module to have procedures getState and setState. The execution of A.getState() in state $\mathbf{m}$ must return the value $f\left(\mathcal{G}_{A}^{\mathbf{m}}\right)$ where $f$ is an arbitrary injective mapping from the type $\mathcal{G}_{A}$ to some parameter type sbits. The setState procedure gets an argument $x:$ sbits and sets $\mathcal{G}_{A}^{\mathbf{m}}$ to $f^{-1}(x)$ if $f^{-1}(x)$ is defined.

Altogether this gives an approach for working with rewindable adversaries in EasyCrypt. See Sec. 4 for details.

\subsection{Rewinding in Other Verification Frameworks}

To the best of our knowledge, rewinding has not been implemented in EasyCrypt, nor in other frameworks for reasoning about cryptographic proofs such as CryptHOL [7] in Isabelle, FCF [17] in Coq, CryptoVerif [9] in OCaml, Verypto [8] in Isabelle, CertiCrypt [6] in Coq, SSProve [1] in Coq.

A natural question is: Can the methods from the present work be ported to these other frameworks? Can rewinding be implemented in those frameworks in other ways? If our work on rewinding in EasyCrypt is any indication, then it is hard to make a reasonable estimate how difficult it is to implement (or even whether it is possible at all) before actually going through the process of doing it. Nonetheless, we will hazard some guesses:

CryptHOL, FCF, Verypto, CertiCrypt, and SSProve are foundational frameworks. That is, they are implemented inside the general purpose logic of existing frameworks such as Coq and Isabelle. This means that the details of the probabilistic semantics of the language are already directly accessible; probabilistic reflection as we did in EasyCrypt would not be necessary. Then it should be possible to implement the different result on reflection (Sec. 3) directly as theorems in Coq/Isabelle. (How hard or easy the proofs of these theorems would be is, like always in formal verificiation, hard to predict in advance.)

On the other hand, CryptoVerif is, like EasyCrypt, not foundational. This means that we cannot directly access the semantics, and thus would probably have to implement something akin to our probabilistic reflection. In addition, it is not clear whether the various facts about rewinding (Sec. 3) can even be expressed inside CryptoVerif. If not, the

\footnotetext{
${ }^{1}$ The restriction to countable types is arbitrary. We could choose a different, larger type sbits instead of bitstrings for encoding program states. However, we choose bitstrings because these are more natural in the computational setting - notions of runtime of algorithms apply naturally to bitstrings but not to uncountable types such as reals.
} 
logic of these tools would have to be extended, and the tools rewritten accordingly.

But, as we stated, these are educated guesses only. To know for sure, one would have to actually put in the work and implement rewinding in these various frameworks.

\section{Preliminaries}

In this section we review the syntax and semantics of the main EasyCrypt constructs. Readers familiar with EasyCrypt can skip this section and just familiarize themselves with our syntactic conventions in this footnote ${ }^{2}$. The top-level definitions in EasyCrypt consist of types, operators, lemmas/axioms, module types, and modules. In EasyCrypt one can specify datatypes and operators, where types intuitively denote non-empty sets of values and operators are typed pure functions on these sets. EasyCrypt provides basic builtin types such as unit, bool, int, etc. The standard library includes formalizations of lists, arrays, finite sets, maps, probability distributions, etc. EasyCrypt also allows users to implement their own datatypes and functions (including inductive datatypes and functions defined by pattern matching). For example, we can give a definition of a polymorphic identity function as follows:

op id ['a]: 'a $\rightarrow$ 'a $=\lambda x . x$.

In this paper, for ease of readability, we use a more compact notation $\lambda \mathrm{x} . \quad \mathrm{x}$ for lambda-abstractions. In the original EasyCrypt code, this would be written as fun $x \Rightarrow x$.

The ambient logic in EasyCrypt is based on a classical (i.e., non-constructive) set theory which we can use to state and prove properties. (The term ambient logic refers to a built-in logic in EasyCrypt. Ambient logic is not specific to reasoning about programs). For example, we can prove that application of id to any $x$ equals to $x$. In lemmas and axioms we will use symbols $\forall$ and $\exists$ instead of the EasyCrypt syntax which uses keywords forall and exists, respectively.

lemma id_prop ['a]: $\forall\left(x:{ }^{\prime} a\right)$, id $x=x$.

proof. trivial. qed.

In EasyCrypt, a proof starts with the keyword proof. The steps of the proof consist of tactic applications (e.g., auto, trivial, etc.) which either discharge the proof obligation or transform it into subgoal(s). The qed finishes the proof.

Types and operators without definitions are abstract and can be seen as parameters to the rest of the development. Parameters can additionally be restricted by axioms. For example, we can parameterize the development by a uniform distribution of elements of type bits.

\footnotetext{
${ }^{2}$ We write $\leftarrow$ for both $<-$ and $<\mathrm{Q}, \stackrel{\$}{\leftarrow}$ for $<\$, \wedge$ for $\wedge, \vee$ for $\backslash /, \leq$ for $<=, \forall$ for forall, $\exists$ for exists, $\mathbf{m}$ for $\& \mathrm{~m}, \mathcal{G}_{A}$ for glob A, $\mathcal{G}_{A}^{\mathbf{m}}$ for (glob A) $\{\mathrm{m}\}$, $\lambda x . x$ for fun $\mathrm{x} \Rightarrow \mathrm{x}, \times$ for $*, \mathrm{t} \mathcal{L}$ for $\mathrm{t}$ list, $\mathrm{t} \mathcal{D}$ for $\mathrm{t}$ distr, $\mu$ for $\mathrm{mu}$, $\mu_{1}$ for mu1, t $O$ for $\mathrm{t}$ option. Furthermore, in $\mathrm{Pr}$-expressions, in abuse of notation, we allow sequences of statements instead of a single procedure call. It is to be understood that this is shorthand for defining an auxiliary wrapper procedure containing those statements.
}

type bits.

op bD : bits $\mathcal{D}$.

axiom bDU : is_uniform bD.

The theory containing this axiom can later be "cloned" and the operator bD instantiated with a value for which the axiom bDU is actually provable. This enables modular design of theories.

In this paper we will use notation bits $\mathcal{D}$ as a more concise version of the EasyCrypt syntax bits distr which denotes the type of distributions of bits. Similarly, we will use bits $\mathcal{L}$ instead of bits $l$ ist, and bits $O$ instead of bits option.

Distributions. In EasyCrypt, every type $t$ is associated with the type $t \mathcal{D}$ of discrete distributions. A discrete distribution over type $t$ is fully defined by its mass function. i.e. by a non-negative function $f$ from elements of type $t$ to reals so that $\Sigma_{x} f(x) \leq 1$. The probability mass function of distribution $\mathrm{d}$ can be accessed by writing $\mu_{1} \quad \mathrm{~d}$ (i.e., $\mu_{1} \quad \mathrm{~d} \quad \mathrm{x}$ is the probability assigned to element $x$ by distribution $d$ ). Also, for any predicate $P \quad: \quad t \rightarrow$ bool we can write $\mu \quad d \quad P$ to get the total probability assigned by $d$ to elements which satisfy $P$.

Modules. In EasyCrypt, modules consist of typed global variables and procedures. The set of all global variables of a module is the union of the set of global variables that are declared in that module and the set of all global variables (declared in other modules) which the module could read or write by a series of procedure calls beginning with a call of one of its procedures. In EasyCrypt, the whole memory (state) of a program is referred to by \&m (or \&n etc.). We can refer to the tuple of all global variables of the module $A$ in \&m as (glob A) $\{m\}$. The type of all global variables of $A$ (i.e., the type of (glob A) $\{m\}$ ) is denoted by glob A. For readability, we will use syntax $\mathcal{G}_{A}$ for the type glob A. Memories \&m will be typed in bold without the \& (i.e., $\mathbf{m}$ for $\& \mathrm{~m}$ ). And $\mathcal{G}_{A}^{\mathbf{m}}$ will denote the EasyCrypt value (glob A) $\{m\}$.

For illustration, we implement the following example of a guessing-game module GG:

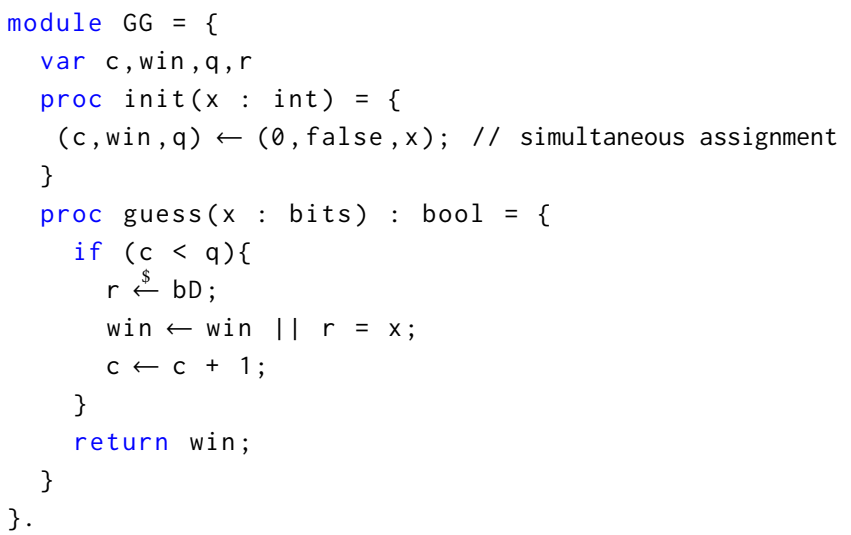


The module GG has three global variables: $\mathrm{c}$ and $\mathrm{q}$ of type int, and w in of type bool. Hence, for any memory $\mathbf{m}, \mathcal{G}_{G G}^{\mathbf{m}}$ has type $\mathcal{G}_{G G}$ which equals to a product bool $\times$ int $\times$ int. The GG module allows a player to guess (call the GG . gues $s$ procedure) the next value sampled from distribution $\mathrm{bD}$. The player has at most $q$ attempts (set during initialization by procedure GG . init). The player wins if they guess correctly at least once.

Module types. In EasyCrypt, module types specify the types of a set of module procedures [4]. Therefore, module types in EasyCrypt are similar to interfaces in other programming languages (e.g., Java). We can specify the module type of $\mathrm{GG}$ as follows:

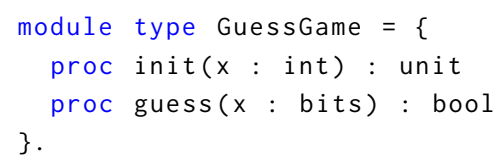

Note that module types say nothing about the global variables a module could have and only specify the input and output types of the module procedures.

Next, we define a module type of protocol parties (adversaries), who receive an instance $G$ of a guessing game as a module parameter. An adversary must have a play procedure which starts the game:

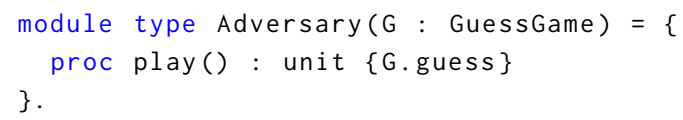

To forbid adversaries to reinitialize the game the play procedure can only execute the guess procedure of the parameter game $\mathrm{G}$. This is optionally expressed by listing the allowed method(s) in the curly braces next to the procedure.

Probability expressions. EasyCrypt has $\mathrm{Pr}$-constructs which can be used to refer to the probabilities of events in program executions: $\operatorname{Pr}[r \leftarrow X \cdot p() @ m: M r]$ denotes the probability that the return value $r$ of procedure $p$ of module $X$ given initial memory $m$ satisfies the predicate M. (I.e., the general form is $\operatorname{Pr}$ [program @ initial memory: event].) The $\mathrm{Pr}$-notation in EasyCrypt is somewhat restrictive, the program can only be a single procedure call. In our presentation, we relax this notation and allow multiple statements; it is to be understood that in the actual EasyCrypt code this is implemented by defining an auxiliary wrapper procedure that contains those statements.

For example, we can express that for any adversary A the probability of winning the guessing-game is equal to or smaller than $\frac{q}{n}$, where $n$ is the size of the support of distribution used by GG and $q$ is the maximal allowed number of guesses.

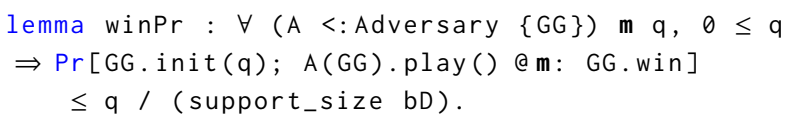

(In EasyCrypt, $\mathrm{X}<$ : $\mathrm{T}$ states that the module $\mathrm{X}$ satisfies the module type T.) Note that the module type Adversary also includes adversaries who simply set the value GG. win to true. EasyCrypt allows us to write Adversary $\{\mathrm{GG}\}$ to denote a subset of adversaries who has disjoint set of global variables from the module GG.

\section{Toolkit for Probabilistic Reflection}

In this section, we discuss a derivation of probabilistic reflection for programs (i.e., modules) in EasyCrypt. Recall that by probabilistic reflection, we mean tools to get access to probabilistic denotational semantics of imperative programs inside EasyCrypt proofs. (Without needing any meta-reasoning.) Also, we use the probabilistic reflection to derive a powerful toolkit of lemmas which are common in pen-and-paper proofs when arguing about distributions underlying programs.

\subsection{Probabilistic Reflection}

Recall that in Sec. 1.2, we introduced Thm. 1.2 that proves the existence of a distribution corresponding to a program's denotational semantics. In EasyCrypt, we formally state this theorem as follows:

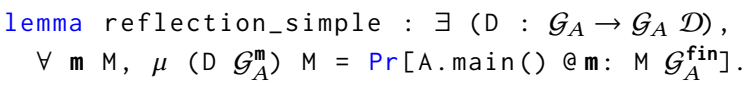

Here, $D$ g corresponds to the family $D_{A}^{g}$ from Thm. 1.2, i.e., D $\mathcal{G}_{A}^{\mathrm{m}}$ is the supposed distribution of final states of the program after running on initial memory $\mathbf{m}$.

Inside an EasyCrypt proof, this lemma could be used as elim (reflection_simple A) $\Rightarrow$ D H_D; this will introduce a variable $D$ in the environment of the proof, together with its defining property $H_{-} D$ stating the relationship between $\mathrm{D}$ and $\operatorname{Pr}[\mathrm{A}$. main ()...].

However, reflection_simple as stated is not general enough for many purposes. In particular, if A. ma in takes an argument $i$ or returns a value $r$ then we cannot reason about the distribution of $r$ and express how $D$ depends on $r$. The following more general reflection lemma removes these limitations:

$$
\begin{aligned}
& \text { lemma reflection : } \exists\left(D: \mathcal{G}_{A} \rightarrow \text { at } \rightarrow\left(r \mathrm{t} \times \mathcal{G}_{A}\right) \mathcal{D}\right), \\
& \quad \forall \mathrm{m} \mathrm{M}, \mu\left(\mathrm{D} \mathcal{G}_{A}^{\mathrm{m}} \text { i }\right) \mathrm{M} \\
& \quad=\operatorname{Pr}\left[r \leftarrow \text { A.main(i) } @ \mathrm{~m}: M\left(r, \mathcal{G}_{A}^{\text {fin }}\right)\right] .
\end{aligned}
$$

The intuition behind this lemma and the previous one is the same. The only difference is that $D$ now has an additional argument $i$, referring to the input of A . main, and the resulting distribution ( $D G_{A}^{\mathrm{m}} \mathrm{i}$ ) is a distribution over pairs $\left(r, \mathcal{G}_{A}^{\text {fin }}\right)$ of output and final memory.

Note that while EasyCrypt allows us to all-quantify over the module A and over the argument and input types (i.e., at and $r t$ ), we cannot quantify over the name ma in of the procedure. Similarly, the number of arguments of procedure ma in is fixed in the lemma. Fortunately, this only constitutes a minor inconvenience, not a real restriction because 
we can always define a wrapper module $A^{\prime}$ that has a procedure main with a single argument $i$ (possibly of a tuple type). Then main can untuple $i$ and invoke the procedure that we actually want to investigate. A simple call to the tactic inline $A^{\prime}$. main in the proof will then unwrap this wrapper procedure.

In EasyCrypt, we directly prove reflection and derive reflection_simple as an immediate corollary. For readability, we proceed with describing a direct proof of reflection_simple instead.

Proof. We start the proof by defining a predicate $\mathrm{P}$ on probabilities which is parameterized by an initial state $g$ of type $\mathcal{G}_{A}$ and an element $x$ of type $\mathcal{G}_{A}$. Below we use the EasyCrypt tactic pose to give a definition which is local to the proof.

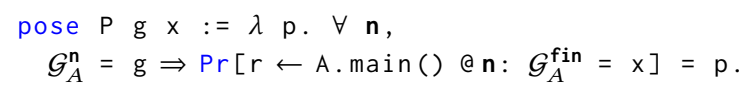

The probability $p$ satisfies the predicate $(P \quad g \quad x)$ if it equals to the probability of $A$. main terminating in the state $x$ by starting its run from a memory $\mathbf{n}$ which has $\mathcal{G}_{A}$ variables equal to $g$.

Before continuing with the proof, we explain that the standard library of EasyCrypt provides the formalization of the Axiom of Choice in the form of the operator choiceb and its corresponding property choi cebP:

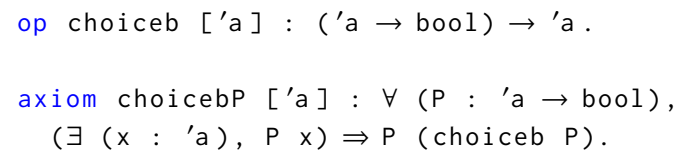

It states that for any predicate $P$ if there exists an element which satisfies it then the element denoted by (choiceb P) satisfies $P$. Here, it is worth mentioning that all propositions in EasyCrypt have type bool.

The next step of the proof is to define a function ( $Q$ g $x$ ) which uses the choice operator on the predicate $(P g x)$ to assign a probability to $x$.

pose $Q \mathrm{~g} x$ := choiceb $(P \mathrm{~g} x)$.

The intuition is that $(Q \mathrm{~g} x)$ returns "the" probability $\operatorname{Pr}\left[\right.$ A.main () @ $\left.\mathbf{n}: \mathcal{G}_{A}^{\text {fin }}=\mathrm{x}\right]$ for all $\mathbf{n}$ with $\mathcal{G}_{A}^{\mathbf{n}}=\mathrm{g}$. Note that a priori we do not know that there is such a probability, because probability could depend on $\mathbf{n}$. To show that ( $Q \begin{array}{ll}Q & \mathrm{x}\end{array}$ ) is a well-defined we need to prove that the value $\left(\begin{array}{lll}Q & g & x\end{array}\right)$ satisfies the predicate $(P \quad g \quad x)$. Because in the lemma, ( $D \mathrm{~g}$ ) is only used for $\mathrm{g}$ of the form $\mathcal{G}_{A}^{\mathrm{m}}$, we specifically need to show the following claim:

have Q_well_def : $P G_{A}^{\mathrm{m}} \times\left(\mathrm{Q} \mathcal{G}_{A}^{\mathrm{m}} \mathrm{x}\right)$.

(Here we use the EasyCrypt tactic have name : fact which allows to locally prove the fact and call it name.)

If we can show that there exists a probability $q$, so that ( $\mathrm{P} \mathcal{G}_{A}^{\mathrm{m}} \times \mathrm{q}$ ) then the proof Q_well_def amounts to a simple application of the choi cebP property. The obvious candidate for this probability $\mathrm{q}$ is the $\mathrm{Pr}$-expression:
$\operatorname{Pr}\left[\right.$ A.main() $\left.@ \mathrm{~m}: \mathcal{G}_{A}^{\mathrm{fin}}=\mathrm{x}\right]$.

To show that this candidate satisfies $\left(P \mathcal{G}_{A}^{\mathrm{m}} \mathrm{x}\right.$ ), we must prove the following (by definition of $\mathrm{P}$ ):

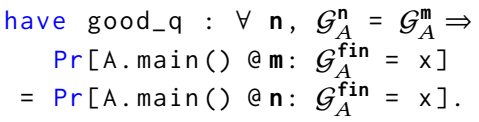

The good_q is intuitively simple and we prove it using the pRHL which is available in EasyCrypt.

Now, as we know that ( $Q \mathrm{~g}$ ) assigns adequate probabilities to elements of type $\mathcal{G}_{A}$, we use the standard EasyCrypt constructor mk which turns any function of type 'a $\rightarrow$ real into distribution of type 'a $\mathcal{D}$.

pose $\mathrm{D} g:=\mathrm{mk}(\mathrm{Q} g)$.

The above defines a parameterized distribution $D$ typed as $\mathcal{G}_{A} \rightarrow \mathcal{G}_{A} \mathcal{D}$. We skip the technical details of a proof which shows that $D$ is a well-formed probability distribution. We only show the final derivation which proves that $D$ is the denotation of A. main. To achieve this we show the pointwise equality of distribution $\mathrm{D}$ and $\mathrm{Pr}$-expression. Let $\mathrm{x}$ be an element of type $\mathcal{G}_{A}$ :

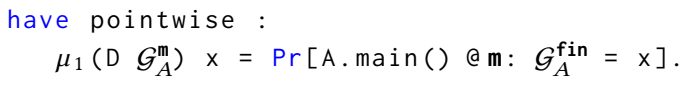

The proof is as follows:

$$
\begin{aligned}
\mu_{1}\left(\mathrm{D} \mathcal{G}_{A}^{\mathrm{m}}\right) \mathrm{x} & =\mu_{1}\left(\mathrm{mk}\left(\mathrm{Q} \mathcal{G}_{A}^{\mathrm{m}}\right)\right) \mathrm{x} \\
& =\mathrm{Q} \mathcal{G}_{A}^{\mathrm{m}} \mathrm{x} \\
& =\operatorname{choiceb}\left(\mathrm{P} \mathcal{G}_{A}^{\mathrm{m}} \mathrm{x}\right) \\
& =\operatorname{Pr}\left[\mathrm{A} . \operatorname{main}() @ \mathrm{~m}: \mathcal{G}_{A}^{\mathrm{fin}}=\mathrm{x}\right]
\end{aligned}
$$

The first equality is by definition of $D$, in the second equality $\mu_{1}$ cancels application of $\mathrm{mk}$ since $\mathrm{D}$ is a well-defined distribution (see muK from EasyCrypt standard library), the third equality is by definition of $Q$, the fourth equality is an application of the previously proved property Q_well_def.

At the first glance, it seems that this implies that ( $D \mathcal{G}_{A}^{\mathrm{m}}$ ) indeed describes the probability distribution corresponding to A.main. That is, we want:

have onsubs : $\mu\left(\mathrm{D} \mathcal{G}_{A}^{\mathrm{m}}\right) \mathrm{M}=\operatorname{Pr}\left[\mathrm{A}\right.$.main()@ $\left.\mathrm{m}: \mathrm{M} \mathcal{G}_{A}^{\mathrm{fin}}\right]$. meaning that the probability that a value sampled from (D $\mathcal{G}_{A}^{\mathrm{m}}$ ) satisfies $M$ equals the probability that the final state of A. main satisfies M. Unfortunately, this is not immediate. For example, hypothetically, the function $M \mapsto$ $\operatorname{Pr}\left[\right.$ A.main () @ m: $M \mathcal{G}_{A}^{\text {fin }}$ ] might not be a discrete probability measure and thus it might not be determined by its values on singleton sets. To show on subs, we need to use one more trick: We define an auxiliary module and procedure $P$. sampleFrom such that $P$. sampleFrom (d) simply returns some $\mathrm{x} \stackrel{\$}{\leftarrow} \mathrm{d}$. Then $\left(\mu\left(\mathrm{D} \mathcal{G}_{A}^{\mathrm{m}}\right) \mathrm{M}\right)$ equals to $\operatorname{Pr}\left[\mathrm{x} \leftarrow \mathrm{P}\right.$. samplefrom $\left.\left(D \mathcal{G}_{A}^{\mathrm{m}}\right) @ \mathrm{~m}: M \mathrm{x}\right]$ and we get:

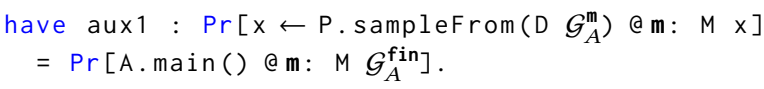


For goals of this shape, we use a combination of the byequi v and bypr tactics from EasyCrypt; byequiv changes this goal into a pRHL judgment relating the programs $A$. main and P. sampleFrom. And bypr converts such a pRHL judgment back to an equality of probabilities. It seems that we are back at onsubs now. However, the final equality is actually:

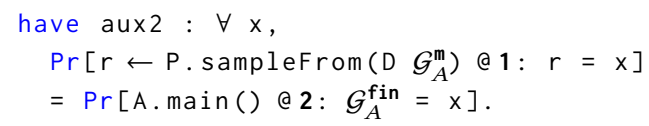

(for memories $\mathbf{1}$ and $\mathbf{2}$ that are equal to $\mathbf{m}$ in global variables of $G_{A}$.) But this follows from poin $t$ wi se proven above.

We clarify that reflection results are proved "once and for all” - by using the EasyCrypt's module cloning mechanism the reflection lemma could be instantiated for arbitrary adversaries.

Note that in our proof we rely on the fact that the tactics byequiv and bypr in combination imply that the probability $\operatorname{Pr}[\ldots: M x]$ (even for infinite $M$ ) can be related to $\lambda y . \operatorname{Pr}[\ldots: x=y]$.

\subsection{Probabilistic Toolkit}

In this section, we present well-known results from probability theory which we formalized and used extensively in our EasyCrypt development. In particular, recall that in Thm. 1.1 we used averaging and Jensen's inequality in the derivation of the key lemma needed for proving the security of a coin-toss protocol (see Sec. 5). In turn, proofs of Jensen's inequality and averaging depend on finite probabilistic approximation. (To the best of our knowledge, none of these results were previously formalized in EasyCrypt.)

Finite Pr-approximation. We prove that the support of a distribution can be finitely approximated with arbitrary precision. We formally prove finite approximation for distributions and then use the probabilistic reflection to extend this result to programs.

Let $d$ be a distribution of type 'a $\mathcal{D}$. Then there exists a sequence of lists $(L \quad n)$ so that the probability that an element sampled from $d$ is not in the list $\left(\begin{array}{l}L \\ n\end{array}\right)$ converges to 0 (for $n \rightarrow \infty$ ). (This holds for discrete distributions only.)

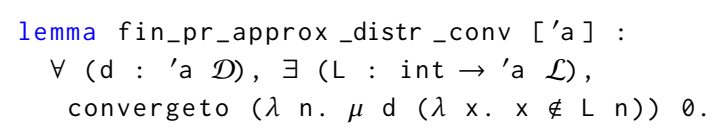

Having the finite probabilistic approximation for distributions allows us to use the probabilistic reflection mechanism to extend the finite probabilistic approximation to programs. More specifically, let A . main be a procedure which takes an argument of type at and produces the result of type $r t$. In this case, there exists a sequence of lists $\left(\begin{array}{ll}L & n\end{array}\right)$, so that the result and the final state produced by A. main ( $i$ ) are not in $\left(\begin{array}{ll}L & n\end{array}\right)$ with probability converging to 0 .

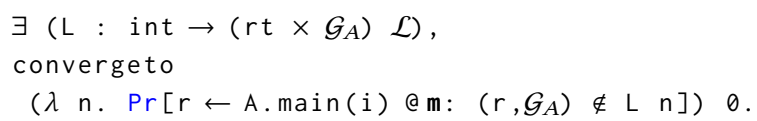

Averaging. Averaging allows us to express the probability corresponding to a program $x \stackrel{\$}{\leftarrow} d$; A. main $(x)$ in terms of probabilities corresponding to the program A. main $(x)$ and the probability assigned to $x$ by distribution $d$. In this sense, the averaging technique can be seen as a generalized version of case-analysis.

In our EasyCrypt formalization we state and prove a general version of averaging for an arbitrary distribution $d$ which also might have an infinite support:

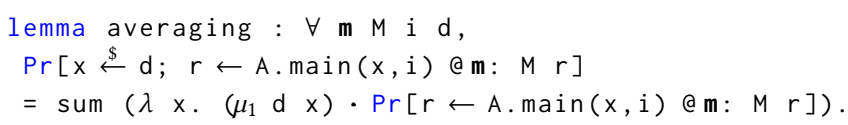

Here, the value (sum $\mathrm{f}$ ) denotes $\Sigma_{x} f(x)$. (Note that the sum in EasyCrypt does not have to range over a finite set.)

Jensen's inequality. Jensen's inequality is another wellknown result which is widely used in cryptography. In general, it relates the value of a convex function of an inte$\mathrm{gral} / \mathrm{sum}$ to the integral/sum of the convex function. In the context of probability theory, it is generally stated in the following form: if $X$ is a distribution, $g$ maps elements of $X$ to reals, and $f$ is convex then $f(E X g) \leq E X(f \circ g)$. Here, $E X h$ is an expected value and $\circ$ denotes function composition.

We prove a slightly restricted version of Jensen's inequality. In particular, we assume that on the support of $X$ the function $g$ takes values in an interval between some parametervalues $a$ and $b$ and that $f x$ takes values in an interval between parameter-values $c$ and $d$ if $a \leq x \leq b$. Also, the standard assumptions are that $f$ is convex, that the distribution $X$ is lossless (i.e., $\mu \times(\lambda \times$. true $)=1)$, and that the expectations $E X g$ and $E X(f \circ g)$ exist.

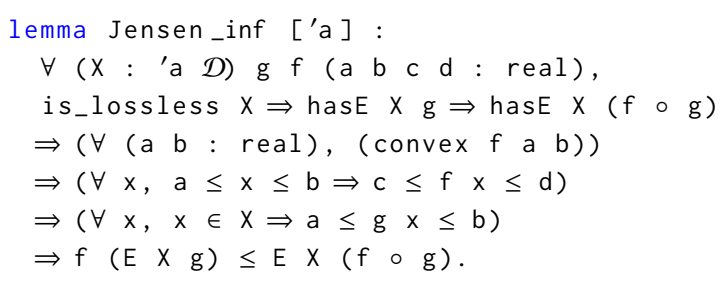

(The EasyCrypt standard library derives Jensen's inequality for distributions with finite support only.)

\subsection{Reflection of Composition}

In this section we address the probabilistic reflection of the sequential composition of programs. For example, let us analyze the program: $r_{1} \leftarrow A \cdot \operatorname{ex}_{1}() ; A \cdot \operatorname{ex}_{2}\left(r_{1}\right)$. We can use the reflection_simple lemma from Sec. 3.1 to get access to a distribution $D_{12}$ such that:

$$
\begin{aligned}
\forall & \mathbf{m} M, \mu\left(D_{12} G_{A}^{\mathrm{m}}\right) \mathrm{M} \\
& =\operatorname{Pr}\left[r_{1} \leftarrow A \cdot \operatorname{ex}_{1}() ; A \cdot \operatorname{ex}_{2}\left(r_{1}\right) @ \mathbf{m}: M \mathcal{G}_{A}^{\text {fin }}\right] .
\end{aligned}
$$


The distribution $\mathrm{D}_{12}$ corresponds to a composite program as a whole. However, being able to reflect the distribution corresponding to a composite program is not enough to enable reasoning about composite programs based on the properties of its components; we do not know how $D_{12}$ is related to $A . e x_{1}$ and $A$. ex 2 separately.

In the following, we prove a lemma for reflection of composition which allows us to show that there exist distributions $D_{1}$ and $D_{2}$ which are the probabilistic reflection of procedures $A$. ex $x_{1}$ and $A . e x_{2}$, and that the composition of $D_{1}$ and $D_{2}$ is $D_{12}$. So, the main goal is to prove lemmas that allow us to derive the distribution of a more complex program from the distributions which correspond to its components.

In EasyCrypt, the composition of distributions is implemented as an operator dlet which has the following type: 'a $\mathcal{D} \rightarrow($ ' $a \rightarrow$ 'b $\mathcal{D}) \rightarrow$ 'b $\mathcal{D}$. Intuitively, the distribution ( dlet $d_{1} d_{2}$ ) could be described imperatively as: $\mathrm{x}_{1} \stackrel{\$}{\leftarrow} \mathrm{d}_{1} ; \mathrm{x}_{2} \stackrel{\$}{\leftarrow} \mathrm{d}_{2} \mathrm{x}_{1} ;$ return $\mathrm{x}_{2}$.

We can formally state the theorem of reflection of composition as follows:

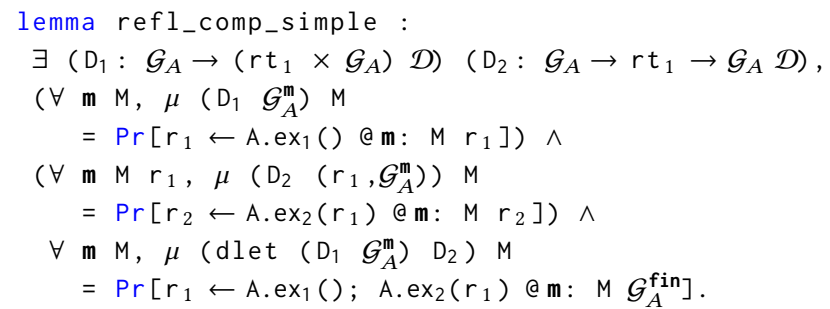

We give only a rough sketch of the proof. First, by using the reflection lemma from Sec. 3.1 we get distributions $D_{1}$ and $D_{2}$ which correspond to procedures A. ex 1 and A. ex 2 , respectively. Next, we use $\mathrm{pRHL}$ reasoning to prove that the imperative composition of $D_{1}$ and $D_{2}$ corresponds to composition of A. ex $x_{1}$ and A. ex $x_{2}$ :

$$
\begin{aligned}
& \operatorname{Pr}\left[x_{1} \stackrel{\$}{\leftarrow} D_{1} ; x_{2} \stackrel{\$}{\leftarrow} D_{2} x_{1} @ m: M x_{2}\right] \\
& =\operatorname{Pr}\left[r_{1} \leftarrow A \cdot \operatorname{ex}_{1}() ; A \cdot \operatorname{ex}_{2}\left(r_{1}\right) @ \mathbf{m}: M \mathcal{G}_{A}^{\text {fin }}\right] .
\end{aligned}
$$

Finally, we prove that the imperative composition of $D_{1}$ and $D_{2}$ corresponds to their declarative composition, namely, dlet $D_{1} \quad D_{2}$ :

$$
\begin{aligned}
& \operatorname{Pr}\left[\mathrm{x}_{1} \stackrel{\$}{\leftarrow} \mathrm{D}_{1} ; \mathrm{x}_{2} \stackrel{\$}{\leftarrow} \mathrm{D}_{2} \quad \mathrm{x}_{1} @ \mathrm{a} \mathrm{m}: \mathrm{M} \mathcal{G}_{A}^{\mathrm{fin}}\right] \\
& =\mu\left(\operatorname{dlet}\left(\begin{array}{lll}
D_{1} & G_{A}^{m}
\end{array} D_{2}\right) M\right. \text {. }
\end{aligned}
$$

This step uses averaging (see Sec. 3.2).

In EasyCrypt formalization we prove a stronger lemma refl_comp which generalizes refl_comp_simple in the following aspects:

- The procedures A. ex 1 and A. ex 2 take all-quantified arguments $i_{1}$ of type $a t_{1}$ and $i_{2}$ of type at $t_{2}$, respectively. As a result, the distributions $D_{1}$ and $D_{2}$ also become parameterized by values of types $a t_{1}$ and $a t_{2}$, respectively.

- The distribution ( $D_{2}$ i $g$ ) is over pairs $\left(r, G_{A}^{\text {fin }}\right)$ of output of A. ex 2 and final memory (not just the final memory).
- In the event part of the probability expression (i.e., $\left.\operatorname{Pr}\left[\ldots: M\left(r_{1}, r_{2}, \mathcal{G}_{A}^{\text {fin }}\right)\right]\right)$ we allow the predicate $M$ to depend on the $r_{1}$ (output of A. ex $)_{1}$ ), $r_{2}$ (output of $A . e x_{2}$ ), and the final memory $\mathcal{G}_{A}^{\text {fin }}$ (not just the final memory).

In EasyCrypt, we prove the following general version of reflection of composition:

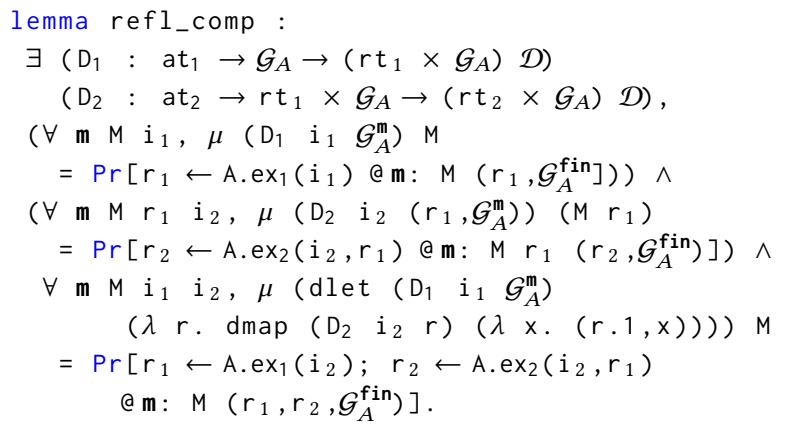

Here, ( $d$ map $d f$ ) denotes the distribution of $(f x)$ for $\mathrm{x} \stackrel{\$}{\leftarrow} \mathrm{d}$.

\section{Rewinding}

In Sec. 1, we briefly explained that rewinding is a commonly used technique which allows one module (i.e., program) to save a state of another module and also restore that state at some later time. More precisely, we say that a module A is rewindable iff:

1. There exists an injective mapping $f$ from $\mathcal{G}_{A}$ to some parameter type sbits ${ }^{3}$.

2. The module A must have a terminating procedure getState, so that whenever A. get $S$ tate is called from the state $g: \mathcal{G}_{A}$, the result of the call must be equal to ( $f g$ ) and the state of A must not change.

3. The module $A$ must have a terminating procedure setState, so that whenever it is given an argument $\mathrm{x}$ : sbits, so that $\mathrm{x}=\mathrm{f} \mathrm{g}$ for some $\mathrm{g}: \mathcal{G}_{A}$ then $A$ must be set into a state $g$.

In EasyCrypt, we start formalizing this definition by defining a module type Rew for rewindable programs:

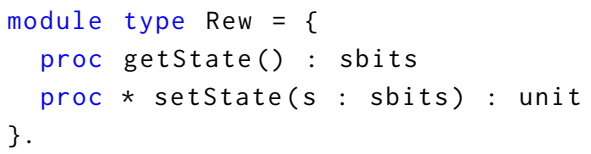

(Here, the symbol * indicates that the procedure (re)initializes all global variables of a module.)

\footnotetext{
${ }^{3}$ Intuitively, sbits is the type of bitstrings. To keep our development more general, we do not require this, but only assume the existence of embeddings nat_sbits: nat $\rightarrow$ sbits (to ensure that sbits is infinite) and pair_sbits: sbits $\times$ sbits $\rightarrow$ sbits. The EasyCrypt theory cloning mechanism makes it possible to later replace this sbits type by a concrete type such as lists of bits.
} 
We formalize the rewinding properties of getState and setState procedures as a predicate RewProp on modules typeable as Rew. Unfortunately, in EasyCrypt we cannot define an operator like RewProp because its definition depends on a module which is not allowed. As a result, in the actual EasyCrypt code the workaround is to copy-andpaste the verbose definition of RewProp (A). This reduces readability, but is conceptually the same.

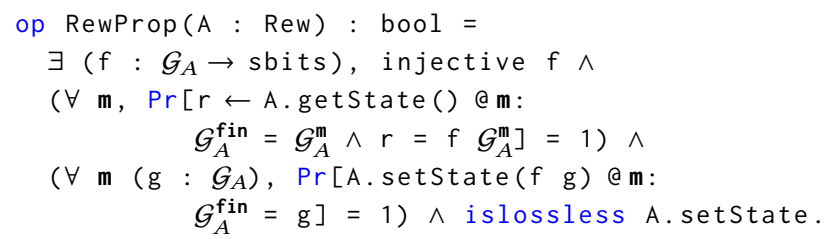

(In EasyCrypt, islossless X.p expresses that the procedure $p$ of module $X$ must terminate on all inputs.)

Then, whenever we do a proof using rewinding, we will need to explicitly assume that our adversary A satisfies property RewProp (A), or, equivalently, quantify only over adversaries of module type Rew satisfying RewProp (A).

The first litmus test of our definition of rewindability is to show that modules without global variables are (trivially) rewindable. For a module without global variables, $\mathcal{G}_{A}$ will be a singleton type (i.e., $\left.\forall\left(\begin{array}{ll}x & y\end{array} \mathcal{G}_{A}\right), \quad \mathrm{x}=\mathrm{y}\right)$. Then we can generically state that $A$ will be rewindable, as long as we implement getState and setState as terminating procedures (it does not matter what they do):

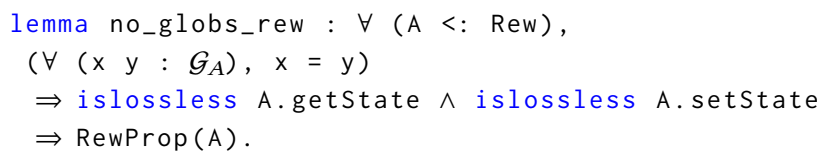

On the necessity of the RewProp axiom. The reader may wonder whether adding the explicit assumption in a security proof that the adversary A satisfies RewProp (A) does not weaken the security proof. After all, it means that security only holds with respect to such adversaries, but not with respect to adversaries that do not satisfy RewProp (A). We argue that RewProp ( $A$ ) is not a true restriction of the adversary, merely a requirement that the adversary has a certain interface with certain properties. The only actual restriction about the inner workings of the adversary that RewProp (A) makes is that the adversary's state can be encoded as a sequence of bits (sbits). Usually, in cryptography, we make even stronger assumptions about the adversary, namely that its state is a sequence of bits (or a Turing machine tape). In contrast, here we only assume that its state can be encoded as a sequence of bits.

We stress that we only need to make this assumption for abstract (i.e., all-quantified) adversaries. For adversaries that we explicitly construct as part of a reduction, we can actually prove RewProp, see the next section.

\subsection{Transformations}

Cryptographic proofs are commonly based on transformations of adversaries (or reduction of adversaries). In EasyCrypt, a transformation is a module which receives other modules as parameters, defines its own global variables, and has procedures which can call procedures of its parametermodules. Typically, one of the parameter-modules will be the original adversary.

In this section, we show how to prove rewindability of a module which is parameterized by rewindable modules and which has at most countable state. We illustrate this by implementing a module $T$ which is parameterized by rewindable modules $A$ and $B$ and has a global variable $x$ of $a$ parameter type $c t$. As a result, the global state of module $T(A, B)$ consist of variable $T . x$ and all global variables of modules A and B. (i.e., $\mathcal{G}_{T(A, B)}=c t \times \mathcal{G}_{A} \times \mathcal{G}_{B}$ ). Since, by the definition of rewindability, we need to embed elements of type $\mathcal{G}_{T(A, B)}$ into sbits then we parameterize our development by an injection from $c t$ to $s b i t s$ :

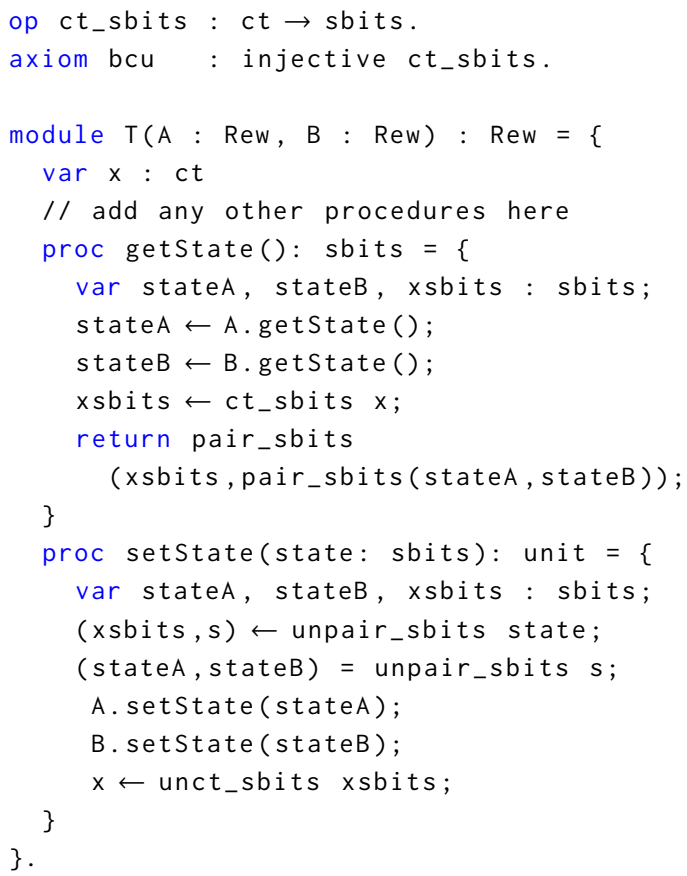

The procedure getState stores the global states of $A$ and $B$ in the local variables stateA and stateB, respectively. Then the global variable $T . x$ is converted into $s b i t s$ and saved in variable $x$ sbits. The resulting state is an embedding of a nested tuple (xsbits, (stateA, stateB)) into sbits. (Recall that pair_sbits is an embedding sbits $\times$ sbits $\rightarrow$ sbits.)

The procedure setState receives an sbits argument which is then "untupled" into sbits variables xsbits, stateA, and stateB. The state of A is set by passing argument stateA to its implementation of set $S$ tate procedure (similarly for $\mathrm{B}$, mutatis mutandis). The variable $\mathrm{T} . \mathrm{x}$ 
is set to the preimage of $x$ sbits. Finally, we use pHL to prove that $T(A, B)$ is also rewindable:

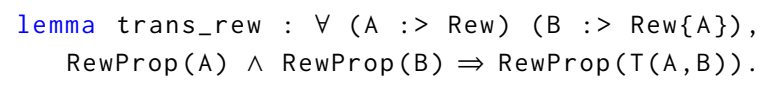

Note, that in the statement of the lemma we additionally require a state of $B$ to be disjoint from a state of $A$ (i.e., $B:>\operatorname{Rew}\{A\})$. This is required because in case of possibly overlapping states not all values of type $\mathcal{G}_{T(A, B)}$ are valid states. For example, if $\mathcal{G}_{A}^{\mathrm{m}}$ and $\mathcal{G}_{B}^{\mathrm{n}}$ have overlapping variables with different values then the value $\left(\mathcal{G}_{A}^{\mathrm{m}}, \mathcal{G}_{B}^{\mathrm{n}}, \mathrm{x}\right)$ is typeable as $\mathcal{G}_{T(A, B)}$ (for any $\mathrm{x}$ of type $\mathrm{ct}$ ), but does not represent a possible state of $T(A, B)$. Unfortunately, in EasyCrypt, it is not possible to express "consistency" of possibly overlapping states of abstract modules.

\subsection{Multiplication Rule and Commutativity}

The multiplication rule from probability theory states that the probability of independent events occurring simultaneously is found by multiplying the probabilities of each event.

In terms of probabilistic programs it is natural to say that an execution of a procedure $P$. run is independent of an execution of $Q$. run if after termination of $P$. r r un the state of $Q$ is not affected. In EasyCrypt, it is easy to prove the multiplication rule for modules with disjoint states:

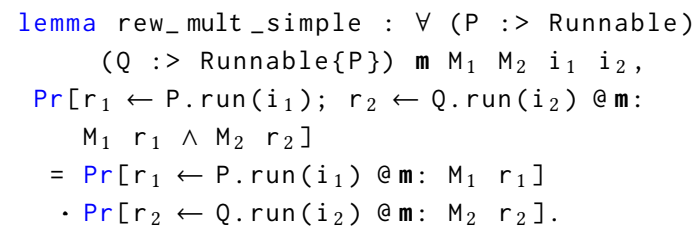

However, if we need independent runs of the procedure(s) of the same module then we need rewinding. Recall, that the main goal of rewindability is to be able to restore the state of a module after running one of its procedures. Let A be a rewindable module (i.e., A satisfies RewProp (A)) with procedures $\mathrm{ex}_{1}$ and $\mathrm{ex}_{2}$ which take all-quantified arguments $i_{1}$ : at $t_{1}$ and $i_{2}: \quad a t_{2}$ and compute results $r_{1}: r t_{1}$ and $r_{2}: r t_{2}$, respectively. Let us analyze the following program.

(1) Save the initial state of A by $s \leftarrow$ A. getState ().

(2) Run the procedure $r_{1} \leftarrow A \cdot \operatorname{ex}_{1}\left(i_{1}\right)$.

(3) Restore the initial state by calling A. setState ( $s$ ).

(4) Run the procedure $r_{2} \leftarrow A \cdot \operatorname{ex}_{2}\left(i_{2}\right)$.

First, we analyze the steps (1)-(3) as a standalone program. In particular we must show that the getState and the set $S$ tate calls do not affect the result computed by $A$. ex $_{1}$ procedure and also show that the final state of $A$ equals to its initial state.

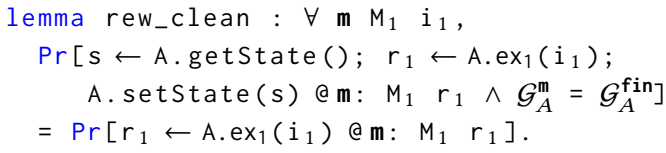

(The proof requires only basic pHL tactics and rewindability axioms.) This result allows us to derive the multiplication rule which states that the probability of a joint event $M_{1} r_{1} \wedge M_{2} r_{2}$ for the program (1)-(4) on memory $\mathbf{m}$ equals to the product of probabilities of events $M_{1} \quad r_{1}$ and $M_{2} \quad r_{2}$ occurring after independent runs of A. ex ${ }_{1}$ and $A . e_{2}$ on $\mathbf{m}$, respectively. In EasyCrypt this is stated as follows:

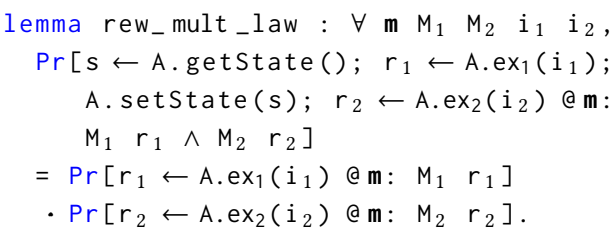

In its essence, rew_mult _law is derived by a single call to the built-in seq tactic.

Commutativity. In its turn, the multiplication rule opens for us an easy route to proving commutativity for rewindable modules. Consider a program consisting of steps (1)-(4)-(3)(2) (i.e., A. ex ${ }_{1}$ and A. ex 2 calls are swapped). We can prove that it computes the same distribution of pairs $\left(r_{1}, r_{2}\right)$ as the program (1)-(4).

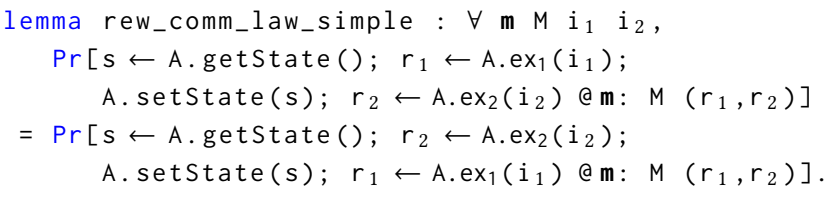

In our formalization by using averaging we prove even stronger version of commitativity for programs which begin with state initializer (see lemma rew_comm_law in file RewCommutes.ec of the accompanying code [15]).

\subsection{Rewinding with Initialization}

In Thm. 1.1 we sketched a derivation of the equation which is needed to prove sum-binding property for commitments (see Sec. 5). More specifically, we analyzed a program which starts with an explicit state initializer, saves the resulting state of module A, runs a procedure A . r un for the first time, restores the saved state, and then runs the $A$. $r$ un procedure for the second time. We proved that the probability of a success (according to some predicate) in two sequential runs of A . run is lower-bounded by a square of probability of a success in a "initialize-then-run" case (i.e., initialize the state and execute the A. run procedure once).

In EasyCrypt, we derive a similar equation, but for a more general case:

- The initialization is done with a procedure $B$. init, where $B$ is a module with a state which can possibly intersect with the state of module $A$.

- The initialization produces a result $r_{0}$ of a parameter type which is then supplied to A. run.

- The procedure B . in it receives all-quantified argument $i$ of a parameter type. 
- The procedure A . run returns a result of a parameter type $r t$. The success of a run is defined by a parameter predicate $M\left(r_{0}, r_{i}\right)$, where $r_{0}$ and $r_{i}$ are the values returned by $B$. init and $A$. run procedures, respectively.

The EasyCrypt statement of the lemma is as follows:

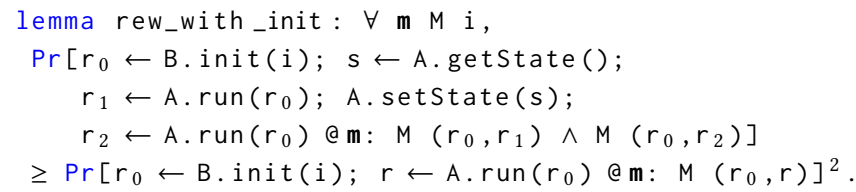

We skip the proof as it roughly follows the steps sketched in Thm. 1.1.

\section{Case Study: Coin-Toss Protocol}

As a case study for our techniques we prove the security of a coin-toss protocol based on bit-commitment. Historically, Blum described the problem of coin-toss protocol with the following example: Alice and Bob are recently divorced, living in two separate cities, and want to decide who gets to keep the car. To decide, Alice wants to flip a coin over the telephone. However, Bob is concerned that if he were to tell Alice the result of his coin toss, she would adjust hers and automatically tell him that she wins. Thus, the problem with Alice and Bob is that they do not trust each other; the only resource they have is the telephone communication channel, and there is not a third party available to read the coin [10].

In the following, we describe the coin-toss protocol based on a bit-commitment scheme which is similar to the original Blum's solution to the coin-toss problem:

1. Alice chooses a random bit $r_{1}$ and then generates a commitment $c$ containing that bit (let $d$ be the respective opening).

2. Alice sends the commitment $c$ to Bob.

3. Bob chooses a random bit $r_{2}$ and sends it to Alice.

4. Alice opens her commitment by sending the bit $r_{1}$ and the opening $d$ to Bob.

5. Bob verifies that $d$ is a valid opening of $r_{1}$ for $c$. Otherwise Bob aborts.

6. Alice and Bob compute the final bit as $r_{1} \oplus r_{2}$ (xor).

The coin-toss protocol must ensure the following property: if at least one of the parties correctly generates a random bit, then the final bit will be (nearly) random.

Security of the coin-toss is almost immediate if the commitment scheme satisfies a property called "sum-binding" in [18]. This property says that the probability of Alice opening the commitment to fal se and the probability of Alice opening it to true add to at most 1 (plus a negligible error). This property in turn is implied by the usual "computationally binding" property which says that Alice cannot open to both false and true simultaneously (except with negligible probability). Showing that "computationally binding" implies "sum-binding", however, requires rewinding. Therefore that proof is a prime candidate for our case-study. (In the post-quantum setting, for example, computationally binding does not imply sum-binding [2]. This illustrates that this seemingly trivial implication is not as easy as it might seem, and that we indeed need rewinding here.)

\subsection{Commitments}

The standard library of EasyCrypt defines the module type Commi tmen $\mathrm{S}$ cheme which requires a scheme $\mathrm{S}$ to implement the following procedures:

1. $\mathrm{p} \leftarrow \mathrm{S}$.gen ( ) generates the public key of a commitment scheme (also known as the public parameters).

2. $(c, d) \leftarrow$ S. commit $(p, m)$ produces commitmentopening pair for a message $m$ and a public key $p$.

3. $b \leftarrow s$. verify $(p, m, c, d)$ returns $b=$ true iff $\mathrm{d}$ is a valid opening for message $\mathrm{m}$, commitment $\mathrm{c}$, and public key $p$.

For our development, we additionally require the existence of a verification function Ver (an "operator" in EasyCryptparlance) which must agree with the procedure S. ver if y on all arguments:

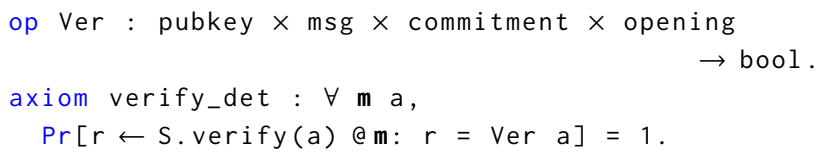

This means that verification is side-effect free (and deterministic). Otherwise, two runs of the verification algorithm could interfere with each other (and with calls to $S$. commit) and give different results.

In cryptography, a commitment scheme is called computationally binding iff the probability that adversary A can produce a commitment with openings of two different messages is negligible. The EasyCrypt standard library defines a module type Binder with a single procedure bind; we can then define the probability of success of adversary A : Binder in the "binding-game":

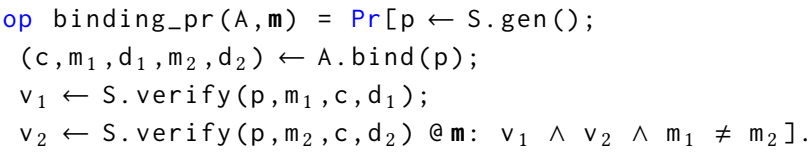

(Here, binding_pr is only a shortcut notation used in this text.) Hence, scheme is binding iff binding_pr $(A, m)$ is negligible for all $A$ and $\mathbf{m}$.

Sum-Binding. Next, we define the "sum-binding" property of commitments. Let $A$ be an adversary and $p_{b}$ be a probability that $A$ can open the commitment to contain $b$ given input $b=f a l s e, t r u e$. The commitment scheme is sumbinding iff for all such adversaries the $\mathrm{p}_{\mathrm{f}}+\mathrm{p}_{\mathrm{t}} \leq 1+\epsilon$, where $\epsilon$ is negligible. We define a module type SumB inder with procedures commit and open. Then we define the probability of success of adversary A : SumBinder in the "sum-binding-game": 


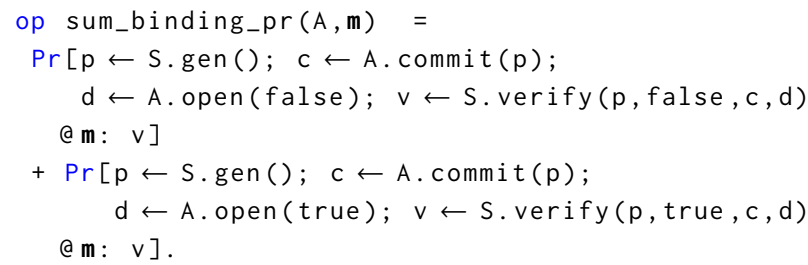

(Again, sum_binding_pr is only a shortcut notation.) Hence, scheme is sum-binding if and only if for all A and $\mathbf{m}$, there exists a negligible $\epsilon$, so that we can show that sum_binding_pr $(A, m) \leq 1+\epsilon$. Before addressing the sum-binding property for commitments, we prove a more generic sum-binding inequality which shows that the sum of probabilities of success of independent runs of arbitrary procedures A. ex 1 and A. ex 2 is related to the probability of joint success in the same run. ${ }^{4}$ More specifically, assume that module $A$ is rewindable and $B$. in it is some initialization procedure. We let $p_{1}$ be the probability that after initialization the procedure $A$. ex ${ }_{1}$ succeeds according to some predicate $M$ (similarly for $\mathrm{p}_{2}$ and $A$. ex $\mathrm{x}_{2}$, mutatis mutandis). In this case, we can prove that the sum of probabilities $p_{1}+p_{2}$ is upper-bounded by a sum $1+2 \cdot q$, where $q$ is the probability that A. ex 1 and A. ex 2 both succeed in the same run (i.e., both starting from the same initial state produced by B . init). In EasyCrypt, we state this equation as follows:

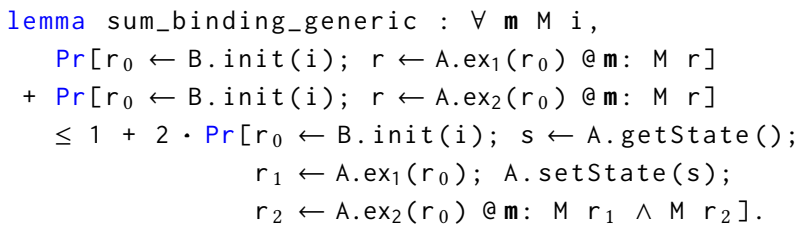

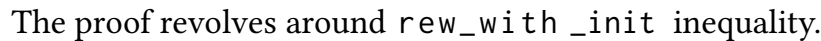

Equipped with the generic sum-binding inequality, we can now finish the proof that binding commitment schemes are also sum-binding. We start by implementing a reduction $R(A)$ which runs $A$. commit (to produce the commitment), stores the state of $A$, runs $A$. open ( $f a l s e$ ) (to produce the first opening), restores the state of $A$, and runs A. open (true) (to produce the second opening). Then we show that the probability that $R(A)$ produces two valid openings (i.e., breaks binding) is lower-bounded in terms of the probability that $A$ is successful in producing one valid opening.

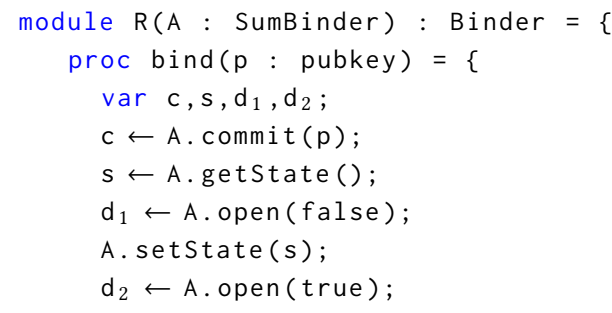

${ }^{4}$ This generic lemma may also be useful when analyzing extractors for proof of knowledge protocols with two challenges, e.g., the zero-knowledge protocols for Hamiltonian cycles [11] and graph isomorphism [12].

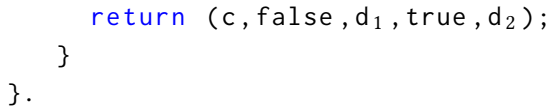

Next, we implement wrapper-modules $B$ and $A^{\prime}$, so that $B$. init is a wrapper around the "commitment initialization" phase $p \leftarrow S$.gen ()$; c \leftarrow A$. commit $(p)$. The procedure $A^{\prime} \cdot e_{1}$ is defined as $A$. open ( $f a l s e$ ), and $A^{\prime}$. ex $x_{2}$ as $A$. open (true). In this case, sum-binding for commitments becomes an immediate consequence of the inequality sum_binding_generic and we can conclude:

lemma commitment_sum_binding : $\forall \mathbf{m}$,

sum_binding_pr $(A, m) \leq 1+2$. binding_pr $(R(A), m)$.

\subsection{Coin-Toss Protocol}

Recall, that a coin-toss protocol is considered secure if it is ensured that if at least one of the parties correctly generates a random bit then the final bit will be (nearly) random.

In the first case, we assume that Alice is honest and Bob is cheating. To simplify this case, we additionally assume that the commitment scheme is perfectly hiding. This means that Bob gets no information about $r_{1}$ after receiving the commitment c. Therefore, if Alice follows the protocol honestly and $r_{1}$ is uniformly random and independent of $r_{2}$ (due to the perfect hiding) then the bit $\left(r_{1} \oplus r_{2}\right)$ is also uniformly random. (The case of cheating Bob does not involve rewinding and is therefore not the focus of this paper.)

In the second case, we are left to show that if Bob honestly follows the protocol, then for any Alice (adversary A : CoinTossAlice) the resulting bit is nearly uniform. Below we assume that module type CoinTossAl i ce requires a module to have procedures commit and toss, where commit produces a commitment $c$, and toss gets a Bob's bit $r_{2}$ as an argument and then computes a bit together with its opening for $c$. We write coin_toss_pr $(A, m, b)$ to denote a probability of $A$ being able to open the commitment to Boolean b.

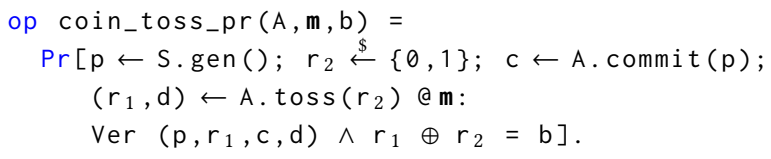

(Once more, coin_toss_pr is only a shortcut notation.) We define $B_{f}(A)$ and $B_{t}(A)$ as the transformations of cointoss adversary into an adversary that breaks binding for the cases $b=$ false and $b=$ true, respectively:

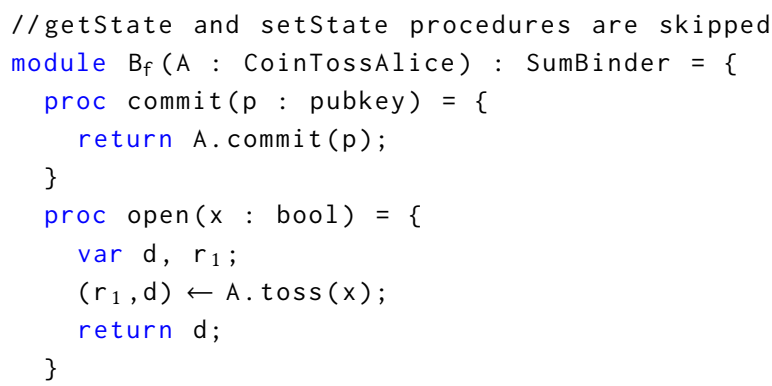




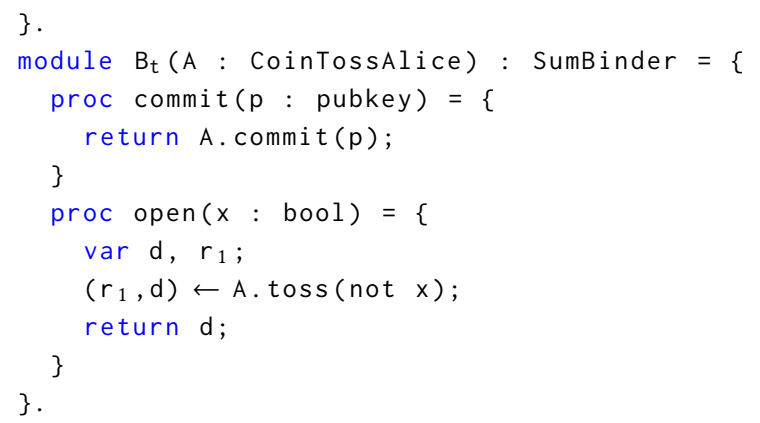

$B_{f}(A)$ delegates the commitment generation to $A$ and when asked to open a commitment to bit $x$ then $x$ is submitted to $A$. toss and the resulting opening is returned. $B_{t}(A)$ is different in that the negation of $x$ is submitted to $A$. tos $s$ Finally, we can derive that if Bob is honest then for any Alice the resulting bit is nearly uniform.

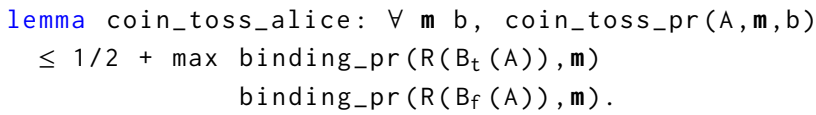

Proof. We start the proof with analysis of the case when $b=$ true (i.e., $r_{1} \oplus r_{2}=$ true). We prove that this case is upper-bounded by $1 / 2+\epsilon$, where $\epsilon$ is the probability of breaking the binding of $S$ by $R\left(B_{t}(A)\right)$.

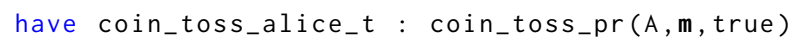

We prove this case by arguing as follows:

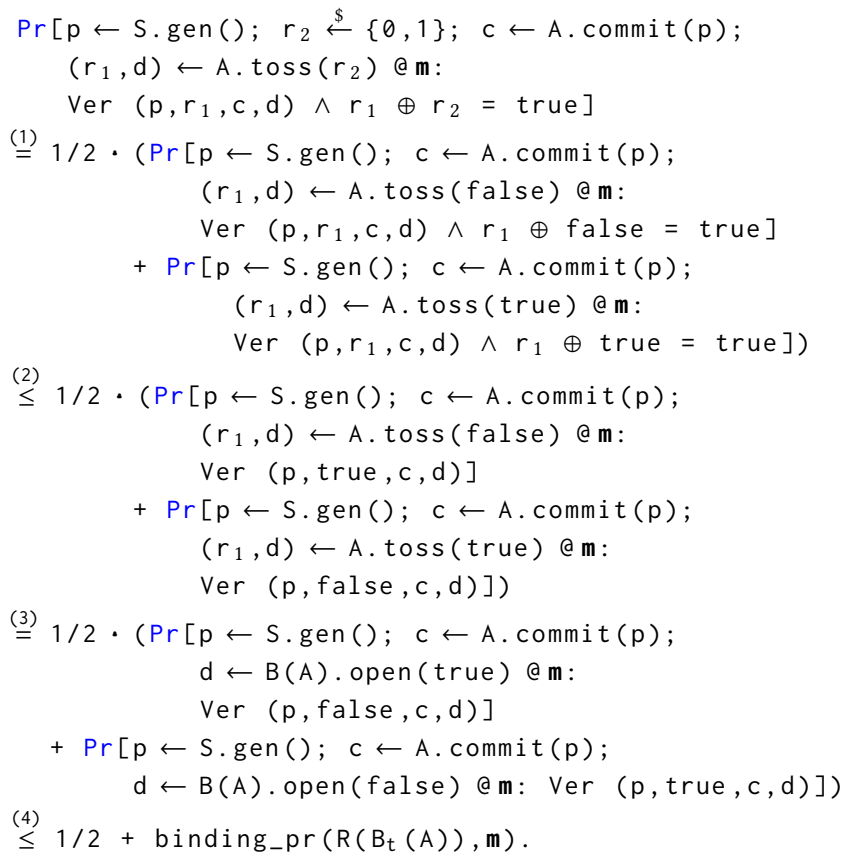

(Here $\{0,1\}$ denotes a uniform distribution of Booleans.) Step (1) is by case distinction of $r_{2}$. Step (2) is by simplification and event-inclusion. Step (3) is by definition of transformation $B_{t}$. Step (4) applies commitment_sum_binding from Sec. 5.1. In a similar way we handle the case when $b=f a l s e$ and show: have coin_toss_alice_f : coin_toss_pr(A,m, false)

$$
\leq 1 / 2+\text { binding_pr }\left(R\left(B_{f}(A)\right), m\right) \text {. }
$$

Finally, coin_toss_alice is a trivial consequence of coin_toss_alice_t and coin_toss_alice_f.

\section{Conclusions}

In this paper we focused on probabilistic reflection and rewindability of adversaries. First, we implemented a powerful toolkit for probabilistic reflection which includes finite probabilistic approximation, averaging, and reflection of composition inside EasyCrypt. Second, we described a notion of rewindable adversaries and derived their basic properties: transformations, multiplication rule, commutativity, rewinding with initialization. Third, by combining these results together we were able to derive a generic sum-binding equation for arbitrary rewindable computations. Fourth, we instantiated the sum-binding property for commitments and proved that if a commitment scheme is binding then it is also sum-binding. Finally, we used this result to prove the security of a bit-commitment based coin-toss protocol.

To the best of our knowledge, probabilistic reflection, rewindable adversaries, and security of a coin-toss protocol have not yet been addressed in theorem provers.

\section{Acknowledgments}

This work was partially supported by the ESF-funded Estonian IT Academy research measure (project 2014- 2020.4.05.190001) and by the ERC consolidator grant CerQuS (819317), by the Estonian Centre of Excellence in IT (EXCITE) funded by ERDF, by PUT team grant PRG946 from the Estonian Research Council.

\section{References}

[1] Carmine Abate, Philipp G. Haselwarter, Exequiel Rivas, Antoine Van Muylder, Théo Winterhalter, Cătălin Hriţcu, Kenji Maillard, and Bas Spitters. 2021. SSProve: A Foundational Framework for Modular Cryptographic Proofs in Coq. In CSF 2021, Vol. 1. IEEE Computer Society, Los Alamitos, CA, USA, 1-15. https://doi.org/10.1109/CSF51468.2021. 00048

[2] Andris Ambainis, Ansis Rosmanis, and Dominique Unruh. 2014. Quantum Attacks on Classical Proof Systems: The Hardness of Quantum Rewinding. In 2014 IEEE 55th Annual Symposium on Foundations of Computer Science. 474-483. https://doi.org/10.1109/FOCS.2014.57

[3] Gilles Barthe, George Danezis, Benjamin Grégoire, César Kunz, and Santiago Zanella-Béguelin. 2013. Verified Computational Differential Privacy with Applications to Smart Metering. In 2013 IEEE 26th Computer Security Foundations Symposium. 287-301. https: //doi.org/10.1109/CSF.2013.26

[4] Gilles Barthe, François Dupressoir, Benjamin Grégoire, César Kunz, Benedikt Schmidt, and Pierre-Yves Strub. 2013. EasyCrypt: A tutorial. In Foundations of security analysis and design vii. Springer, 146-166.

[5] Gilles Barthe, Benjamin Grégoire, Sylvain Heraud, and Santiago Zanella Béguelin. 2011. Computer-aided security proofs for the working cryptographer. In Annual Cryptology Conference. Springer, 71-90.

[6] Gilles Barthe, Benjamin Grégoire, and Santiago Zanella Béguelin. 2009. Formal certification of code-based cryptographic proofs. In Proceedings 
of the 36th annual ACM SIGPLAN-SIGACT symposium on Principles of programming languages. 90-101.

[7] David A. Basin, Andreas Lochbihler, and S. Reza Sefidgar. 2020. CryptHOL: Game-based proofs in higher-order logic. Journal of Cryptology 33, 2 (2020), 494-566.

[8] Matthias Berg. 2013. Formal verification of cryptographic security proofs. Ph.D. Dissertation. Saarland University. https://doi.org/10.22028/D29126528

[9] Bruno Blanchet. 2008. A computationally sound mechanized prover for security protocols. IEEE Transactions on Dependable and Secure Computing 5, 4 (2008), 193-207.

[10] Manuel Blum. 1983. Coin flipping by telephone: a protocol for solving impossible problems. ACM SIGACT News 15, 1 (1983), 23-27.

[11] Manuel Blum. 1987. How to prove a theorem so no one else can claim it. In In: Proceedings of the International Congress of Mathematicians. 1444-1451.

[12] Manuel Blum, Paul Feldman, and Silvio Micali. 1988. Non-Interactive Zero-Knowledge and Its Applications. In Proceedings of the Twentieth Annual ACM Symposium on Theory of Computing (Chicago, Illinois, USA) (STOC '88). Association for Computing Machinery, New York, NY, USA, 103-112. https://doi.org/10.1145/62212.62222
[13] Véronique Cortier, Constantin Cătălin Drăgan, François Dupressoir, Benedikt Schmidt, Pierre-Yves Strub, and Bogdan Warinschi. 2017. Machine-checked proofs of privacy for electronic voting protocols. In 2017 IEEE Symposium on Security and Privacy (SP). IEEE, 993-1008.

[14] Denis Firsov, Henri Lakk, and Ahto Truu. 2021. Verified MultipleTime Signature Scheme from One-Time Signatures and Timestamping. In 2021 IEEE 34th Computer Security Foundations Symposium (CSF). IEEE Computer Society, Los Alamitos, CA, USA, 653-665. https: //doi.org/10.1109/CSF51468.2021.00051

[15] Denis Firsov and Dominique Unruh. 2021. Reflection, Rewinding, and Coin-Toss in EasyCrypt - accompanying EasyCrypt code. https: //github.com/dfirsov/easycrypt-rewinding/tree/cpp2022. https://doi. org/10.5281/zenodo.5760917

[16] Jakob Nussbaumer. 2019. Security analysis for IPsec with EasyCrypt. Master's thesis. University of Bonn.

[17] Adam Petcher and Greg Morrisett. 2015. The foundational cryptography framework. In International Conference on Principles of Security and Trust. Springer, 53-72.

[18] Dominique Unruh. 2016. Computationally Binding Quantum Commitments. In Advances in Cryptology - EUROCRYPT 2016, Marc Fischlin and Jean-Sébastien Coron (Eds.). Springer Berlin Heidelberg, 497-527. 